Research Article

\title{
Serological profile of acute undifferentiated fever cases attending a tertiary care hospital: emergence of scrub typhus in Telangana State
}

\author{
Md Khaleel $^{1}$, Md Wajid ${ }^{2}$ \\ ${ }^{1}$ Dr. Md Khaleel, Professor, ${ }^{2}$ Dr. Md Wajid, Assistant Professor, both authors are affiliated with Department of \\ Microbiology, Deccan College of Medical Sciences, Hyderabad., Telangana, India.
}

Address for Correspondence: Dr. Md Khaleel, Professor, Department of Microbiology, Deccan College of Medical Sciences, Hyderabad. Email id: docmic175@gmail.com

\begin{abstract}
Introduction: Present study was undertaken to elucidate aetiology of acute undifferentiated fevers and prevalence of scrub typhus among patients admitted in our hospital. Materials \& Methods: 100 consecutive serum samples received during the period May 2016 to October 2016 were tested for dengue NS1 antigen by ELISA, chikungunya Ig M antibodies by MAC- ELISA, widal tube agglutination test, leptospira $\mathrm{Ig} \mathrm{M}$ antibodies by ELISA, plasmodium falciparum, plasmodium vivax, by Parahit F and V, Weil Felix tube agglutination test, orientiatsusugamushi IgM and IgG antibodies by Immunochromatography. Results: Out of hundred samples subjected to more extensive analysis, 39 samples were positive for Dengue which included 28 samples positive for IgM antibodies, 6 samples were positive for IgM antibodies and NS1 antigen, 5 positive for NS1 antigen. 19 were found to be positive for antibodies to orientiatsusugamushi by Immunochromatography. Conclusion: Though the dengue fever is often considered and investigated by the physicians in all such cases, other infections are often ignored or do not figure in the differential diagnosis particularly during outbreaks. There is a need to investigate for scrub typhus, an acute febrile illness caused by Orientia tsusugamushi (Ricketssia tsusugamushi) which is a re-emerging disease in India.
\end{abstract}

Key-words: Acute fevers, Dengue, Scrub typhus, Weilfelix

\section{Introduction}

Dengue fever and scrub typhus are common infections in Asia that often present as acute febrile illness of unclear aetiology. Though the dengue fever is often considered and investigated by the physicians in all such cases, other infections are often ignored or do not figure in the differential diagnosis particularly during outbreaks. There is a need to investigate for scrub typhus, an acute febrile illness caused by Orientia tsusugamushi (Ricketssia tsusugamushi) which is a reemerging disease in India. However Scrub typhus should also be differentiated from malaria, arboviral infections, leptospirosis, and typhoid fever [1]. The distribution of disease corresponds to distribution of vector Leptothrombidium. Endemic foci are associated with specific habitats such as rice fields, river banks and grassy fields [2]. In India rickettsial diseases have been documented from North and South India [3]. Out breaks

Manuscript received: $24^{\text {th }}$ December 2016

Reviewed: $2^{\text {nd }}$ January 2017

Author Corrected: $10^{\mathrm{th}}$ January 2017

Accepted for Publication: $18^{\text {th }}$ January 2017 of scrub typhus has been reported in cooler months in states of Tamilnadu and Pondicherry [4]. Reports of scrub typhus from Andhra Pradesh are scanty [5]. Early diagnosis of bacterial infections like leptospirosis and scrub typhus would be useful in initiating specific antibiotic therapy and prevention of mortality and morbidity. Present study was undertaken to elucidate etiology of acute undifferentiated fevers and prevalence of scrub typhus among patients admitted to tertiary care hospital.

\section{Materials and Methods}

The study was conducted at Deccan Institute of Medical Sciences, Hyderabad during May 2016 to October 2016. Serum samples from 238 patients admitted in our hospital with acute undifferentiated febrile illness, provisionally diagnosed as dengue fever were tested for dengue IgM antibodies by MAC-ELISA (Panbio). A subset of 100 consecutive serum samples received 


\section{Research Article}

during the period May 2016 to October 2016 were further tested for dengue NS1 antigen by ELISA(Panbio), Chikungunya Ig $\mathrm{M}$ antibodies by MAC- ELISA(Panbio), Widal tube agglutination test(Span diagnostics), LeptospiraIg $\mathrm{M}$ antibodies by ELISA (Panbio), Plasmodium falciparum, Plasmodium vivax by Parahit $\mathrm{F}$ and V (Span diagnostics), Weil Felix tube agglutination test (Tulip diagnostics), Orientia tsusugamushi IgM and IgG antibodies by Immunochromatographic test (SD bioline). All the tests were performed according to manufacturer's instructions. Demographic and clinical data of all the patients was recorded. Basic laboratory studies were performed, including a complete blood count, creatinine, glucose, liver function tests, and chest X-ray. An informed consent was taken from all the subjects who were included in the study. The study was approved by institutional ethical committee. The Statistical analysis was performed using SPSS software for Windows version 16.0

\section{Results}

Seventy two (30.37\%) of the 238 serum samples tested were found to be positive for dengue Ig M antibodies. Out of 100 samples subjected to more extensive analysis, 39 samples were positive for Dengue which included 28 samples positive for IgM antibodies, 6 samples were positive for IgM antibodies and NS1 antigen, 5 positive for NS1 antigen. 19 were found to be positive for antibodies to Orientia tsusugamushi by Immunochromatography and Weil Felix test. The Weil felix antibody titres were $\geq 1$ in 320 dilutions in 14, 1 in 160 dilutions in 4, and 1 in 80 dilutions in one sample. Chikungunya Ig M antibodies, Leptospira Ig M antibodies, Widal test, Plasmodium RDT test were positive in 4,9,1 and 7 samples respectively. Mean age for Dengue fever and Scrub typhus was 20.8 years and 36.2 years respectively. Male and female ratio was 1.3:1 among Dengue fever and 1:1.2 among scrub typhus cases. All the scrub typhus fever cases were from rural areas belonging to surrounding districts of Nalgonda, Medak, and Mahboobnagar.

Table-1: Frequency of positivity of various serological tests performed.

\begin{tabular}{|c|c|c|}
\hline Serological test for antibodies & Number & \% \\
\hline Dengue positive & 39 & $39 \%$ \\
\hline Orientia Tsusugamushi +VE BY ICT TEST & 19 & $19 \%$ \\
\hline Weil Felix TEST +VE & 19 & $19 \%$ \\
\hline Chikungunya +VE & 4 & $4 \%$ \\
\hline Leptospira +VE & 9 & $9 \%$ \\
\hline Widal test +VE & 1 & $1 \%$ \\
\hline Plasmodium RDT +VE & 7 & $7 \%$ \\
\hline
\end{tabular}

\section{Discussion}

Most of the acute fevers presenting with rash and thrombocytopenia are presumptively diagnosed as dengue fever, especially during monsoon and post monsoon months in tropical countries like India. However only up to one third of the cases are confirmed as dengue. In our study after extensive serological evaluation, specific etiological diagnosis could be established in $79 \%$ of the cases studied. Even after including NS1 antigen test, dengue fever contributed to $39 \%$ whereas other infections contributed to $40 \%$ of cases and $21 \%$ cases remained undiagnosed.

In India, the presence of scrub typhus has been known for several decades. During world war II scrub typhus produced considerable mortality and morbidity among troops deployed in South East Asia. However in later years the disease virtually disappeared; probably because of wide spread use of insecticides to control other vector borne diseases. Recent reports from several parts of country including South India indicate that there is a re- emergence of scrub typhus. Scrub typhus is grossly underdiagnosed in India due to its nonspecific clinical presentation, low index of suspicion among clinicians and lack of diagnostic facilities.

Diagnosis of scrub typhus is most often confirmed by serological testing. Specific gold standard tests like Immunoflourescence antibody test (IFA), Immunoperoxidase test (IP) and ELISA are not available in our country and isolation of organism in animals and cell culture is limited by lack of containment facilities and expertise in handling the high risk group pathogens. Many reports from Indian subcontinent are based on clinical findings and relatively insensitive and nonspecific Weil Felix test [6]. Weil Felix test is based 
on detection of antibodies to $O X K$ strain of Proteus mirabilis. Criteria suggested for diagnosis of scrub typhus include a single titre of 1:320 or four fold rise in antibody titre from 1:80 in Weil Felix test. Agglutinating antibodies are detectable from 5-10 days following starting of symptoms, with antibody being mainly IgM type, a good correlation between results of Weil Felix test and detection of antibodies by IFA is often observed in diagnosis of scrub typhus in India [3]. In our study antibody titre was $1: 320$ in 14 cases $(73.7 \%)$ and low titre of 1:80-1:160 were detected in 5 cases. Rising antibody titre could not be demonstrated as we could not collect the convalescent sera. In a study conducted in South India at a break point of 1:80 titre, sensitivity was $30 \%$ and the specificity and positive predictive value was found to be $100 \%$ [7].

Sera from $95 \%-99 \%$ of patients with scrub typhus recognize a $56 \mathrm{KDA}$ protein of Orientia tsutsugamushi which comprises of $10-15 \%$ of the total Ricketssial cellular protein content [8]. Recently commercial rapid detection kits employing 56KDA recombinant antigen for detection of $\operatorname{IgM} \& \operatorname{IgG}$ antibodies in acute phase sera have appeared in the market with reported sensitivity of $97-99 \%$ and specificity up to $99 \%$ in the diagnosis of acute scrub typhus fever of $2-10$ days duration $[8,9]$. In the present study $100 \%$ co-relation was seen between Weil Felix test and the rapid diagnostic tests (RDT). None of the sera that were positive for Weil Felix and RDT showed cross reacting antibodies to other infectious agents tested.

\section{Conclusion}

In this study scrub typhus contributed to $19 \%$ of the acute fevers and $41.3 \%$ of undiagnosed fever. To the best of our knowledge except for a case report, there are no reports on the prevalence of scrub typhus in Telangana. This study emphasizes the need for the general awareness of Ricketssial infections in Telangana. Rapid diagnostic tests using 56 KDA antigens of Orientiatsutsugamushiare easily available and provide a cost effective alternative diagnostic tool. More research is essential regarding the epidemiology, pathogenesis and lab diagnosis of this disease in Indian context particularly in Telangana.
Funding: Nil, Conflict of interest: None initiated, Permission from IRB: Yes

\section{References}

1. Mahajan SK. Scrub typhus. J Assoc Physicians India. 2005 Nov;53:954-8.

2. Sharma S, Rakesh K, Kaore SN, Yadav VK, Sharma R. Geographical distribution, effect of season and life cycle of Scrub typhus. JK Sci. 2010;12:63-4.

3. Mahajan SK, Kashyap R, Kanga A, Sharma V, Prasher BS, Pal LS. Relevance of Weil-Felix test in diagnosis of scrub typhus in India. J Assoc Physicians India. 2006 Aug;54:619-21.

4. Vivekanandan M, Mani A, Priya YS, Singh AP, Jayakumar S, Purty S. Outbreak of scrub typhus in Pondicherry. J Assoc Physicians India.2010Jan;58:24-8.

5. Boorugu H, Dinaker M, Roy ND, Jude JA. Reporting a case of scrub typhus from Andhra Pradesh. J Assoc Physicians India. 2010 Aug;58:520.

6. Chogle AR. Diagnosis and treatment of scrub typhus-the Indian scenario. J Assoc Physicians India. 2010 Jan; 58:11-2.

7. Isaac R, Varghese GM, Mathai E, J M, Joseph I. Scrub typhus: prevalence and diagnostic issues in rural Southern India. Clin Infect Dis. 2004 Nov 1;39 (9):1395-6.

8. Ching WM, Rowland D, Zhang Z, Bourgeois AL, Kelly D, Dasch GA, Devine PL. Early diagnosis of scrub typhus with a rapid flow assay using recombinant major outer membrane protein antigen (r56) of Orientia tsutsugamushi. Clin Diagn Lab Immunol. 2001 Mar; 8 (2):409-14.

9. Lijuan Z, Si H, Yuming J, Liang L, Xuemei L, Lianying L, Huilan Y, Qiang Y, Chuangfu C, Shiwen W. A rapid, sensitive and reliable diagnostic test for scrub typhus in China. Indian J Med Microbiol. 2011 Oct-Dec;29(4):368-71. doi: 10.4103/0255-0857.90166.

\section{How to cite this article?}

Md Khaleel, Md Wajid. Serological profile of acute undifferentiated fever cases attending a tertiary care hospital: emergence of scrub typhus in Telangana State. Trop J Path Micro 2017;3(1):52-54.doi: 10.17511/jopm.2017.i1.09. 